\title{
The use of functional magnetic resonance imaging techniques in the evaluation of patients with disorders of consciousness: a case report
}

\author{
Katarzyna Gębska-Kośla ${ }^{1 A, B, C, D, E, F, G}$, Andrzej Głąbiński2 ${ }^{B, D, F}$, Magdalena Sabiniewicz ${ }^{2 F}$, Tomasz Wolak ${ }^{3 B, C, D}$, \\ Mariusz Wachowski ${ }^{1 \mathrm{E}}$, Lidia Kruczykowska ${ }^{4 \mathrm{~B}, \mathrm{~F}}$, Agata Majos ${ }^{1 \mathrm{AB}, \mathrm{B}, \mathrm{D}, \mathrm{F}, \mathrm{G}}$ \\ 'Department of Radiological and Isotopic Diagnosis and Therapy, Medical University of Lodz, Poland \\ 2Department of Neurology and Stroke, Medical University of Lodz, Poland \\ ${ }^{3}$ World Hearing Centre, Institute of Physiology and Pathology of Hearing, Kajetany, Nadarzyn, Poland \\ ${ }^{4}$ Department of Cranio-Maxillofacial and Oncological Surgery, Medical University of Lodz, Poland
}

\section{Abstract}

Purpose: The management of patients with disorders of consciousness (DOC) constitutes a challenge for clinicians.

Case report: We present the case of a 66-year-old man who developed coma with subsequent DOC after a severe traumatic brain injury. Behavioural assessment constitutes the gold standard in the evaluation of patients with DOC. In the case presented herein the neuropsychological findings were ambiguous, and the patient underwent functional magnetic resonance imaging (fMRI) to determine whether he was in a vegetative state or minimally conscious state. Three paradigms: passive, active, and resting state fMRI were used to study the brain activity in our patient.

Conclusions: fMRI provided reliable evidence of preserved minimal consciousness. The neuroimaging techniques used in our patient were vital for his further treatment.

Key words: minimally conscious state, coma, functional MRI.

\section{Introduction}

Evaluation of comatose patients who develop disorders of consciousness (DOC) constitutes a challenge for clinicians. Major disorders of consciousness in comatose patients can be classified into three types: the patient may stay in coma, or the coma may evolve into intermediate states, unresponsive wakefulness syndrome (UWS)/vegetative state (VS), or minimally conscious state (MCS). Although behavioural assessment is the gold diagnostic standard in this setting, it provides only indirect evidence of the consciousness level, which, according to many authors, may lead to misdiagnosis. Based solely on the results of subjective clinical observations, it is often impossible to unequivocally conclude whether a patient with DOC has retained a minimal level of consciousness or not [1]. This distinction, however, is crucial from the clinicians' perspective, because to be able to effectively participate in the rehabilitation treatment, the patient needs to be aware of him/herself and the environment.

Currently available advanced neuroimaging techniques, i.e. functional magnetic resonance imaging, may play a vital role in clinical assessment and have become an additional diagnostic tool providing more precise information about the brain function of patients with DOC [1].

We present the case of a 66-year-old man who developed coma and subsequent DOC after a severe traumatic injury of the brain.

Correspondence address:

Dr. Mariusz Wachowski, Department of Radiological and Isotopic Diagnosis and Therapy, Medical University of Lodz, 251 Pomorska St., Lodz, Poland, e-mail: mariuszwachosky@gmail.com

Authors' contribution:

A Study design · B Data collection · C Statistical analysis · D Data interpretation · E Manuscript preparation · F Literature search · G Funds collection 


\section{Case report}

A 66-year-old male patient was referred to the Neurology Department because of progressing DOC. Two years earlier, the patient experienced a multiple trauma with severe injury of the brain after a motor vehicle accident.

Computed tomography (CT) performed after the accident demonstrated massive subarachnoid bleeding, subdural haematoma in the left frontoparietal area, and intraparenchymal haemorrhage in the right thalamus and left frontal lobe. The injuries resolved slowly within a few weeks. A follow-up magnetic resonance imaging (MRI) performed a month after the accident showed residual haemorrhage areas, up to $10 \mathrm{~mm}$ in diameter, in the corona radiata of both hemispheres and right thalamus, along with small volumes of blood in the left parietal and right temporal lobe sulci. Moreover, the evidence of oedema was found in the body of corpus callosum. At the time of the evaluation, the patient suffered from DOC, did not respond to stimulation, opened his eyes spontaneously, and showed bilateral Babinski reflex, a sign of corticospinal tract damage.

Three months after the accident, the patient could breathe independently and had no injuries that required further treatment. After a percutaneous endoscopic gastrostomy (PEG), the man was transferred to a therapeutic care centre.

\section{Material and methods}

\section{Electroencephalography results and neuropsychologic opinion obtained four months before the functional magnetic resonance imaging and confirmed at the time of the examination}

During the electroencephalography (EEG), the patient was without verbal and logical contact. The EEG record was spatially differential. Basic activity detected in the parietooccipital region included an irregular alpha rhythm (8-10 Hz) with up to $50 \mu \mathrm{V}$ amplitude, mixed with theta waves and faster rhythms. The record contained a small number of disseminated small theta waves. Additionally, a few sharp waves and complexes of sharp and slow waves were detected in the left hemisphere, primarily in the frontoparietal region. The record did not change significantly after photostimulation, except for a burst of activity in the form of the waves described above (Figure 1).

The neuropsychological evaluation revealed neither a reflex landmark nor purposeful movements. The patient was unable to establish verbal or eye contact and opened his eyes spontaneously. The responses to commands were inconsistent. Sporadically, the patient opened and closed his mouth, straightened his arms, and crossed his legs. The pain reflexes were preserved. Reflexive laughter and crying were observed. Sleep-wake cycle was normal.

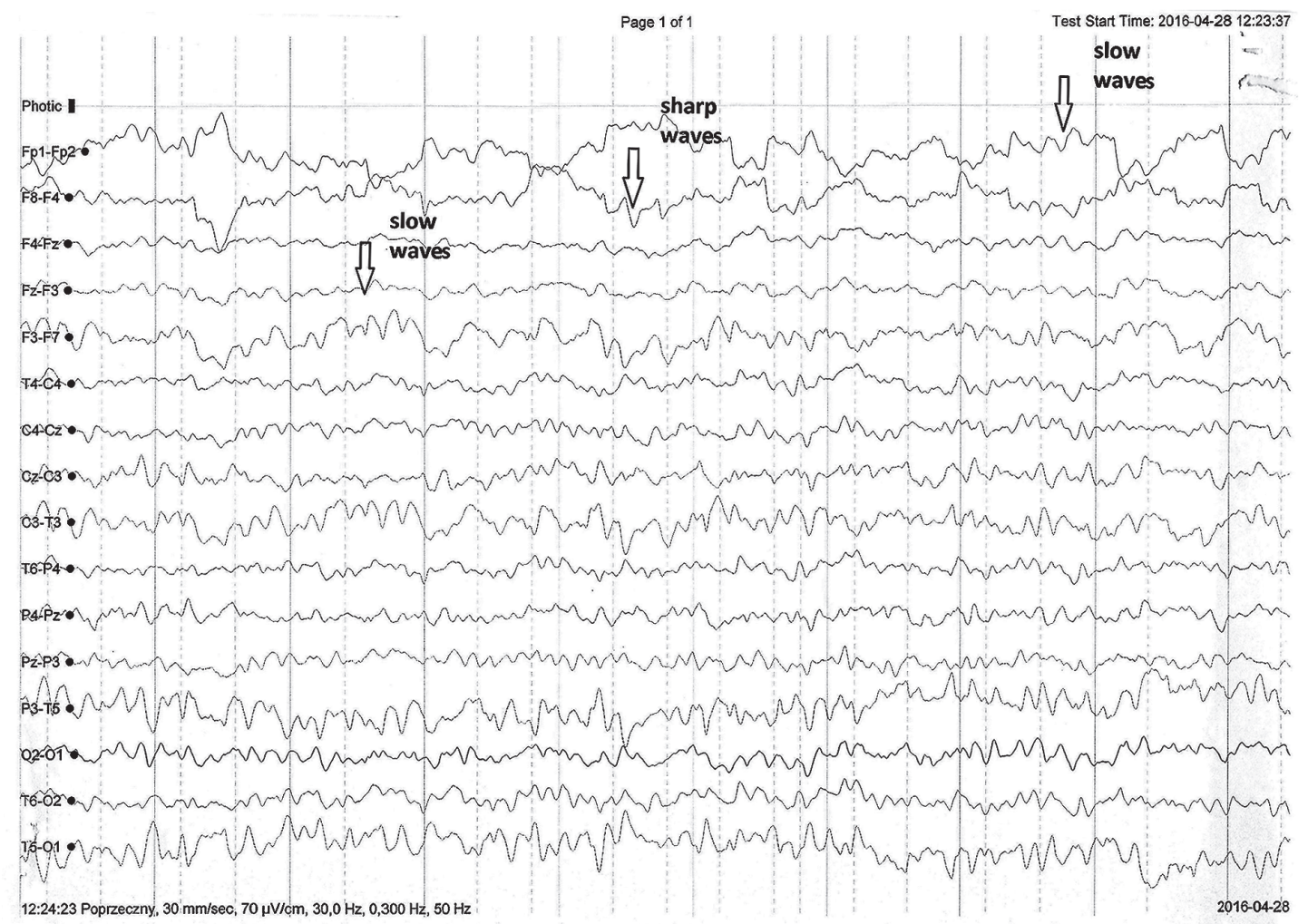

Figure 1. Electroencephalography record: slow theta waves in both hemispheres. Sharp waves observed predominantly in the left frontal and parietal areas. Arrows indicate representative sharp waves and theta waves 
The patient scored 10 points on the Glasgow Coma Scale. Based on the results of clinical tests it was impossible to evaluate whether the patient was in a vegetative state or minimally conscious state. Further follow-up was recommended.

\section{Neuroimaging techniques}

Four different functional magnetic resonance imaging (fMRI) paradigms were used to evaluate the patient's consciousness level [2]. Considering the lack of evidence for visual fixation or pursuit, external auditory stimuli were applied. The examination included one active and three passive fMRI tasks to detect wilful modulations of brain activity. The block-designed study was chosen considering its high detection power.

Each paradigm was divided into five blocks of 10 acquisitions each. The ABABABABAB block design was used, with $A$ representing the baseline activation and B corresponding to the stimuli (each one lasting $30 \mathrm{~s}$ ).

- Church bells. A passive paradigm. During the stimulation periods, the sound of church bells was played followed by white noise during the non-stimulation periods.

- Family member voice. A passive paradigm. During the periods of stimulation, the patient's family member was played to him, and white noise was played in the non-stimulation periods.

- Animal sounds. A passive paradigm. During the periods of stimulation, the sounds of various animals were played, followed by white noise during the non-stimulation periods.

- Applause. An active paradigm. Before the examination, the patient was asked to imagine that he claps his hands each time he hears applause. Then, the applause sound was played during the periods of stimulation, followed by white noise during the non-stimulation periods.

Moreover, a resting state (RS) fMRI was carried out to examine the patient's brain activity at rest.

\section{Functional magnetic resonance imaging data}

The fMRI was carried out with a $1.5 \mathrm{~T}$ scanner (Siemens Avanto Tim $76 \times 18$ ). Morphological, three-dimensional T1-weighted sequences were obtained according to the following protocol: $\mathrm{FOV}=241 \times 218 \mathrm{~mm}$, matrix $=232$ $\times 256, \mathrm{TR}=1160 \mathrm{~ms}, \mathrm{TE}=2.54 \mathrm{~ms}, \mathrm{TA}=2{ }^{\prime 2} 2$. Each acquired volume contained 192 slices with $0.9 \mathrm{~mm}$ thickness.

The functional examination was based on echoplanar imaging (EPI) sequences: $\mathrm{TR}=3000 \mathrm{~ms}, \mathrm{TE}=50 \mathrm{~ms}$, FOV $=192 \times 192 \mathrm{~mm}$, matrix $64 \times 64, \mathrm{TA}=5^{\prime} 11$, with 38 slices, each $3 \mathrm{~mm}$ thick.

The resting state sequences were obtained according to the following protocol: $\mathrm{FOV}=192 \times 192 \mathrm{~mm}$, matrix $=64 \times 64, \mathrm{TR}=2500 \mathrm{~ms}, \mathrm{TE}=50 \mathrm{~ms}, \mathrm{TA}=10^{\prime} 9$, with 32 slices, each $3.5 \mathrm{~mm}$ thick.
The data were analysed with statistical software: SPM 12 and Conn, running in MATLAB. The threshold of statistical significance was set at $p=0.05$. A $t$-value was calculated for each voxel. All voxels with the T-scores above the threshold value were considered active voxels. Further analysis of activated brain regions was based on a large body of evidence from behavioural studies, available neuroimaging and electrophysiology literature, and our own specific hypotheses [2]. The number of activated voxels in clusters (ke) and the values of $t$-statistics for the intensity of activation $(\mathrm{T})$ were determined for the examined regions of interest.

\section{Results}

\section{Passive and active functional magnetic resonance imaging paradigms}

- Church bells. An activation was observed in the head of the right caudate nuclei $(T=6.14$; ke $=284)$ (Figure 2). This part of the nuclei was previously shown to be associated with cognitive and emotional processing [3]. Activation of caudate nuclei was observed in humans presented with a reward [4]. However, according to some researchers, the activation in response to negative stimuli was stronger than after a positive or neutral stimulation [5]. The responses to a negative stimulation were shown to be also associated with additional activation in the ventrolateral prefrontal cortex [5]. Our patient presented with activation in the medial prefrontal cortex $(\mathrm{T}=6.3 ; \mathrm{ke}=209)$, an area responsible for hedonic evaluations (anticipation, appraisal, and memory of pleasurable stimuli) [6] (Figure 3).

- Family member voice. In this paradigm, an activation ( $\mathrm{T}=4.12 ; \mathrm{ke}=300)$ in the left Wernicke area, involved in understanding of a spoken language [7], was observed (Figure 4).

- Animal sounds. No statistically significant changes were observed in this paradigm.

- Applause. The stimulation caused an activation in the supplementary motor area (SMA) $(T=2.4$; ke = 167). According to literature, this type of activation is typical for patients who were asked to imagine a movement [8] (Figure 5).

\section{Resting state functional magnetic resonance imaging}

The test revealed activation in the default mode network (DMN); namely 1) left lateral parietal cortex (LLPC), 2) right lateral parietal cortex (RLPC), 3) medial prefrontal cortex in both hemispheres (MPFC), and 4) posterior cingulate cortex (PCC) in the left hemisphere (Figure 6).

\section{Discussion}

Our patient opened his eyes spontaneously, showed reflexive responses, and had normal sleep-wake cycles. 

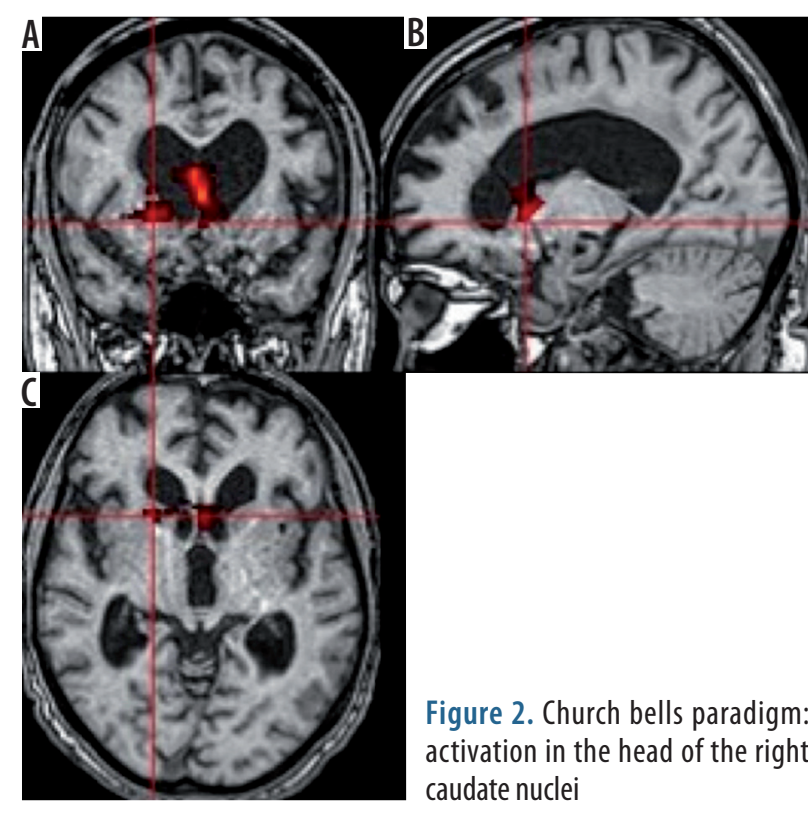

Figure 2. Church bells paradigm: activation in the head of the right caudate nuclei
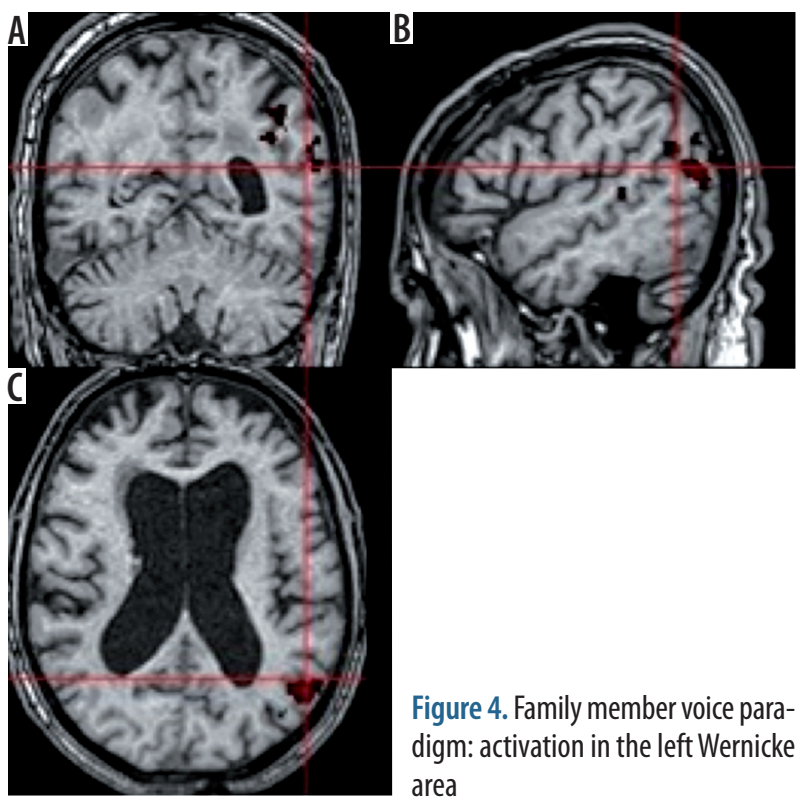

Figure 4. Family member voice paradigm: activation in the left Wernicke area

Such clinical presentation is characteristic for UWS/VS [9]. However, according to his family members, the patient's condition deteriorated shortly before referral to our centre, which was also reflected by the results of neuropsychological assessment.

A few months before the referral, the patient still showed some signs of consciousness, sporadically responded to commands, but was incapable of functional object use and unable to give a gestural/verbal yes/no response. Based on this clinical presentation, his condition was classified as MCS [10]. However, considering his ability to comprehend language, his condition could also be subcategorised as MCS+ [11].

EEG showed moderate changes in the left hemisphere, primarily in the frontoparietal region, with a burst activity the location of which was consistent with the injured area of the brain.
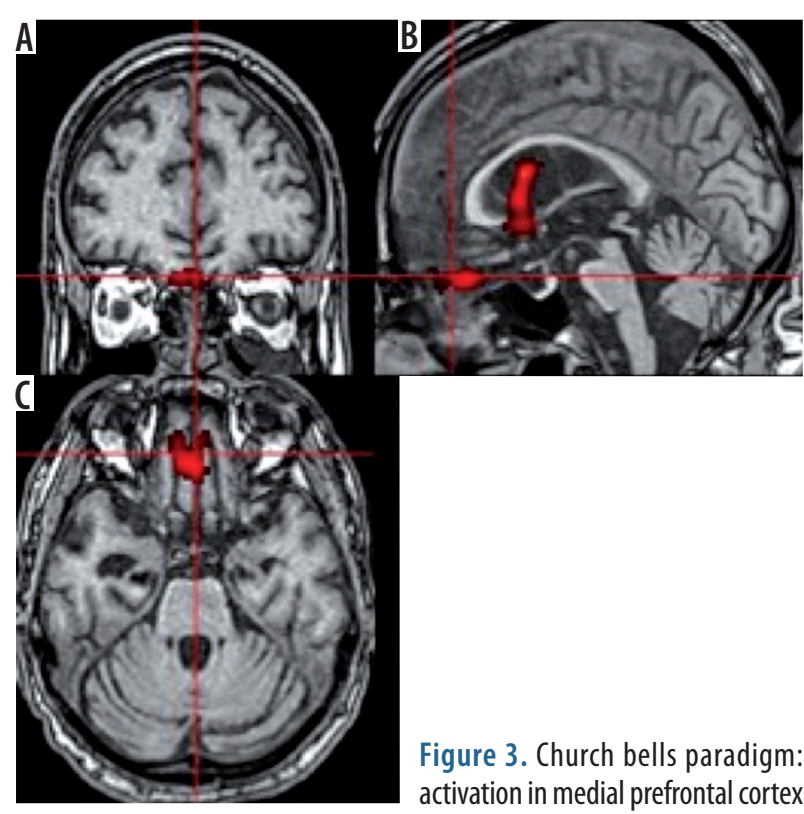

Figure 3. Church bells paradigm: activation in medial prefrontal cortex
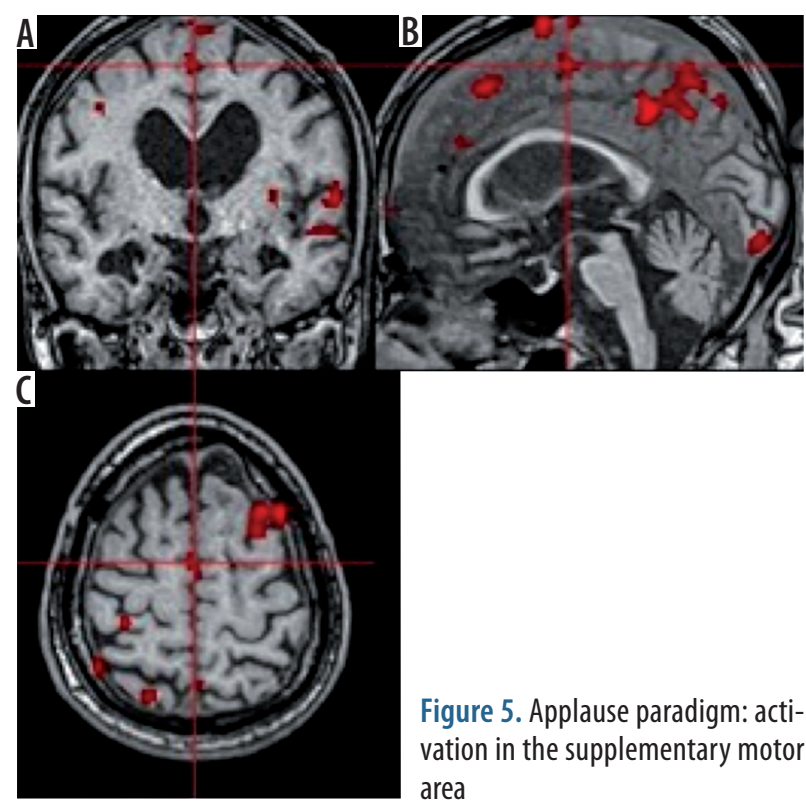

Figure 5. Applause paradigm: activation in the supplementary motor area

Considering an ambiguous result of the most recent neuropsychological evaluation, the neurologists decided to conduct some neuroimaging tests to obtain a better insight into the patient's condition.

Both passive and active fMRI paradigms require the patient's involvement and focus. Hence, we decided to perform those sequences in the first place, during the initial part of the MRI protocol, to avoid potential fatigue. The resting state sequence requires no involvement aside from being awake and therefore was performed at the very end, after approximately a 10-minute interval. The patient was not subjected to any external stimuli during the time of the resting state sequences or during the interval. In the case presented herein, three different passive paradigms were used. Two of them, "church bells" and "family member voice", caused activation within specific regions of the brain, similar to that ob- 

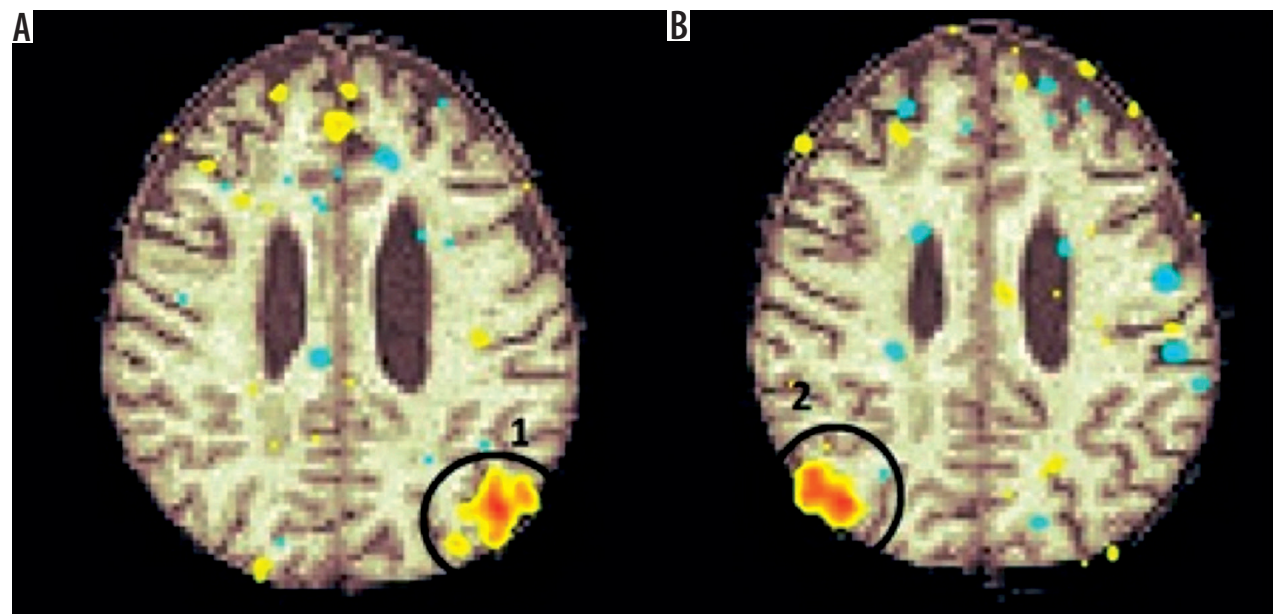

c

D

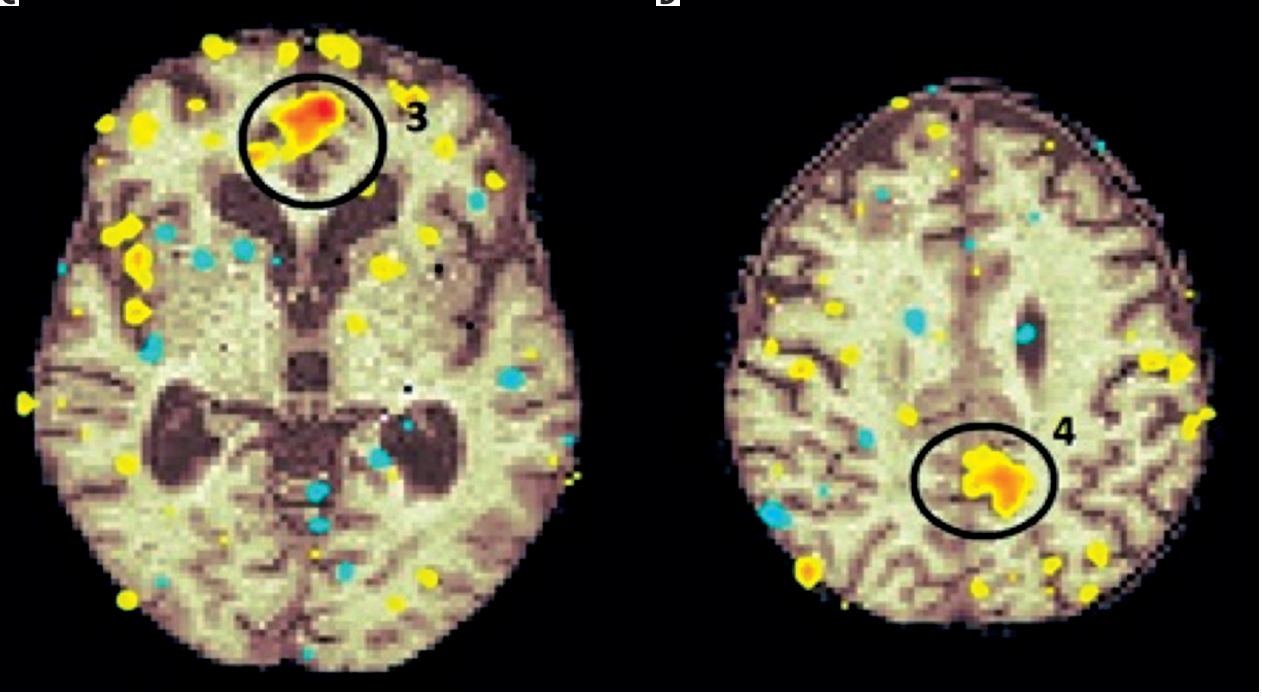

Figure 6. Resting state functional magnetic resonance imaging: activation in the default mode network: 1) left lateral parietal cortex, 2) right lateral parietal cortex, 3) medial prefrontal cortex, 4) posterior cingulate cortex

served in persons with retained consciousness [12]. As our patient was religious before the accident and still showed some behavioural responses to sermons audition after the event, we expected the "church bells" paradigm to act as a strong emotional stimulus. Indeed, a significant activation in the head of the right caudate nuclei and medial part of the fronto-orbital cortex was observed. Activation of both these regions was shown to be associated with pleasure and reward $[3,6]$, which implies that the paradigm might have been a form of positive stimulation.

The use of the "family member voice" paradigm resulted in an activation in the left Wernicke area, a region responsible for understanding spoken language. Consequently, the fMRI confirmed that the patient was able to distinguish between non-language and language auditory stimuli [13]. According to literature, this type of activation is characteristic for MCS [14].

To the best of our knowledge, only a few cases of patients with DOC, who were asked to perform a cognitive task, e.g. imagining swimming or playing tennis, have been published thus far. In our opinion, this might be an argument for uniqueness of the hereby presented case [15]. During fMRI, our patient was asked to imagine hand clapping, which resulted in an activation of the supplementary motor area. This activation pattern was consistent with those reported previously in healthy controls and patients with altered consciousness $[8,15]$. The activation of the supplementary motor area, detected despite the absence of a behavioural evidence of awareness, corresponds to active cognitive processing [16]. Hence, the fMRI proved that our patient was able to understand commands and to respond to them appropriately.

Recently, the term "non-behavioural MCS" $\left(\mathrm{MCS}^{*}\right)$ has been proposed to describe the condition of patients without behavioural signs of consciousness, but who still show consistent activation of the brain, performing various tasks during neuroimaging studies [17]. This is important information from both clinicians' and family members' perspectives because this term could be used to identify patients who may still benefit from pharmacological and electrophysiological therapies [18]. 
Many previous fMRI studies demonstrated the presence of spontaneous brain activity at rest in the form of specific patterns, referred to as resting state (RS) activity [19]. RS fMRI acquisitions are easy to perform because the examination requires neither a stimulation equipment nor the patient's collaboration. Furthermore, the results are believed to be translated easier and faster into clinical practice in patients with DOC. The default mode network $(\mathrm{DMN})$ is a network of specific brain regions that are active in persons who are awake and at rest. Functional connectivity of all DMN areas was shown to be non-linearly correlated with consciousness level, reaching peak values in healthy subjects and persons with locked-in syndrome, and deteriorating gradually in patients with MCS, UWS/ VS, and brain death [20].

In the case presented herein, the DMN activation was similar to that observed in healthy subjects, which implies that our patient might suffer from MCS. We found functional connectivity in the lateral parietal cortex (LLPC, RLPC) and bilateral activation in the medial prefrontal cortex (MPFC). The only area of the brain with unilateral, left-sided activation was the posterior cingulate cortex (PCC).

Posterior cingulate cortex is a key structure for arousal and awareness, and as such, it shows high metabolic activity and functional connectivity in persons with normally retained consciousness [21]. Previous studies demonstrated a structural connectivity between the PCC, thalamus, and brainstem arousal system, and activation of the thalamus and PCC was shown to correlate with the severity of DOC [22].

We found only a few published RS fMRI studies of trauma patients who experienced MCS with brain damage $[23,24]$. In those studies, no DMN activation was observed in the damaged parts of the brain. According to the literature, even partially retained activity of the brain might be sufficient to maintain a certain level of consciousness.

As our patient had a bleeding to the right thalamus after the accident, we suspected that this might be a reason behind the lack of PCC activation in the right hemisphere, and perhaps also a causal factor of DOC.

With no doubt, the condition of our patient has worsened over time. While a few months prior to the referral, the patient's status was classified as MCS+, only limited behavioural signs of consciousness could be observed on admission to our centre. Nevertheless, evidence from neuroimaging studies suggests that the patient was still aware of himself and the environment. This information was vital in the context of his further rehabilitation plan, as the prognosis in patients with MCS (even MCS*) is often better than in those with UWS/VS [25,26]. Furthermore, to be admitted to a rehabilitation centre, the patient needs to be able to participate in the treatment.

Based on this finding, the patient was qualified for further treatment at a coma rehabilitation centre; however, shortly thereafter, he developed pneumonia, which progressed to sepsis and eventually contributed to his demise.

Although fMRI was shown to be of utmost importance in our patient, we are well aware of all the potential limitations of this technique. The exact sensitivity and specificity of fMRI are still unknown, especially if various paradigms are used. Also, some methodological drawbacks exist. The result of fMRI may be affected by motor artefacts, and the response to stimuli may be delayed in some patients due to impairment of cognitive function [10]. All these issues should be considered during interpretation of fMRI data. However, bearing in mind that behavioural assessment, still being the gold standard in the evaluation of patients with DOC, may lead to misdiagnoses, an additional diagnostic tool is needed.

\section{Conclusions}

fMRI provides a novel insight into the brain function of patients with DOC. The neuroimaging techniques used in our patient provided the information that turned out to be crucial for his further treatment. While the result of the neuropsychological evaluation was inconclusive, the neuroimaging study confirmed unequivocally that the patient retained a minimal consciousness, and because of that, he was qualified for further treatment at a coma rehabilitation centre.

The results of fMRI add considerably to clinical assessment, which may improve both diagnosis and prognosis in patients with DOC.

\section{Conflict of interest}

The authors declare no conflict of interest.

\section{References}

1. Giacino JT, Schnakers C, Rodriguez-Moreno D, Kalmar K, Schiff N Hirsch J. Behavioral assessment in patients with disorders of consciousness: gold standard or fool's gold? Prog Brain Res 2009; 177: 33-48.

2. Laureys S, Peigneux P, Goldman S. Brain imaging. In: D'haenen H. den Boer JA, Willner P (eds.). Biological Psychiatry. John Wiley \& Sons Ltd., New York 2002: 155-166.
3. Castellanos FX, Giedd JN, Eckburg P, et al. Quantitative morphology of the caudate nucleus in attention deficit hyperactivity disorder. Am J Psychiatry 1994; 151: 1791-1796.

4. Balleine BW, Delgado MR, Hikosaka O. The role of the dorsal striatum in reward and decision-making. J Neurosci 2007: 27: 81618165 . 
5. Carretié L, Ríos M, De la Gándara BS, et al. The striatum beyond reward: caudate responds intensely to unpleasant pictures. Neuroscience 2009; 164: 1615-1622.

6. Kringelbach ML, Berridge KC. The functional neuroanatomy of pleasure and happiness. Discov Med 2010; 9: 579-587.

7. Fiez JA, Raichle ME, Balota DA, Tallal P, Petersen SE. PET activation of posterior temporal regions during auditory word presentation and verb generation. Cereb Cortex 1996; 6: 1-10.

8. Ehrsson HH, Geyer S, Naito E. Imagery of voluntary movement of fingers, toes, and tongue activates corresponding body-part-specific motor representations. J Neurophysiol 2003; 90: 3304-3316.

9. Laureys S, Celesia GG, Cohadon F, et al. Unresponsive wakefulness syndrome: a new name for the vegetative state or apallic syndrome. BMC Med 2010; 8: 68.

10. Giacino JT, Ashwal S, Childs N, et al.. The minimally conscious state: definition and diagnostic criteria. Neurology 2002; 58: 349-353.

11. Bruno MA, Vanhaudenhuyse A, Thibaut A, Moonen G, Laureys S. From unresponsive wakefulness to minimally conscious PLUS and functional locked-in syndromes: recent advances in our understanding of disorders of consciousness. J Neurol 2011; 258: 1373 1384.

12. Vogel D, Markl A, Yu T, Kotchoubey B, Lang S, Müller F. Can mental imagery functional magnetic resonance imaging predict recovery in patients with disorders of consciousness? Arch Phys Med Rehabil 2013; 94: 1891-1898.

13. Rodd JM, Davis MH, Johnsrude IS. The neural mechanisms of speech comprehension: fMRI studies of semantic ambiguity. Cereb Cortex 2005; 15: 1261-1269.

14. Di HB, Yu SM, Weng XC, et al. Cerebral response to patient's own name in the vegetative and minimally conscious states. Nuerology 2007; 68: 895-899.

15. Laureys S, Schiff ND. Coma and consciousness: paradigms (re) framed by neuroimaging. Neuroimage 2012; 61: 478-491.
16. Edlow BL, Giacino JT, Wu O. Functional MRI and outcome in traumatic coma. Curr Neurol Neurosci Rep 2013; 13: 375.

17. Vogel D, Markl A, Yu T, et al. Can mental imagery functional magnetic resonance imaging predict recovery in patients with disorders of consciousness? Arch Phys Med Rehabil 2013; 94: 1891-1898.

18. Giacino JT, Whyte J, Bagiella E, et al. Placebo-controlled trial of amantadine for severe traumatic brain injury. N Engl J Med 2012; 366: 819-826.

19. Nir Y, Hasson U, Levy I, et al. Widespread functional connectivity and fMRI fluctuations in human visual cortex in the absence of visual stimulation. Neuroimage 2006; 30: 1313-1324.

20. Cauda F, Micon BM, Sacco K, et al. Disrupted intrinsic functional connectivity in the vegetative state. J Neurol Neurosurg Psychiatry 2009; 80: 429-431.

21. Leech R, Sharp DJ. The role of the posterior cingulate cortex in cognition and disease. Brain 2014; 137: 12-32.

22. Fernández-Espejo D, Soddu A, Cruse D, et al. A role for the default mode network in the bases of disorders of consciousness. Ann Neurol 2012; 72: 335-343.

23. Soddu A, Vanhaudenhuyse A, Demertzi A, et al. Resting state activity in patients with disorders of consciousness. Funct Neurol 2011; 26: $37-43$.

24. Noirhomme Q, Soddu A, Lehembre R, et al. Brain connectivity in pathological and pharmacological coma. Front Syst Neurosci 2010; 4: 160 .

25. Thibaut A, Bruno MA, Ledoux D, Demertzi A, Laureys S. tDCS in patients with disorders of consciousness: sham-controlled randomized double-blind study. Neurology 2014; 82: 1112-1118.

26. Luauté J, Maucort-Boulch D, Tell L, et al. Long-term outcomes of chronic minimally conscious and vegetative states. Neurology 2010; 75: 246-252. 